\title{
Heat transfer in 3D water and ice basins
}

\author{
S. Ceci, L. De Biase \& G. Fossati \\ University of Milano Bicocca, Department Environmental Sciences, Italy
}

\begin{abstract}
Studying heat transfer in 3D water or ice basins involves the solution of Navier Stokes or Stokes systems of PDE, coupled with a scalar equation for thermal energy transport.

The method used in this paper is based on a Finite Volume technique where physical quantities and boundary conditions are approximated by means of high order formulae and the time advance is dealt with by a fractional step technique. This paper is mainly concerned with applications, but, with respect to a preceding version of the method, an evaluation of performance of techniques dealing with the turbulent viscosity in water basins is presented and a study of the temperature field in a glacier is included. The method is applied to a thermal discharge in a water basin of lower temperature and to a portion of the Priestley Glacier (Antarctica).

The results are very accurate and coherent with the physical theory and with measured data.
\end{abstract}

Keywords: fluid dynamics, glacier modelling, glacier temperature, thermal discharges.

\section{Brief description of the method}

In this paper some interesting applications of a method presented in Deponti et al. [1] aimed at modelling meso-scale mass and energy flows in glaciers are presented, together with applications of a similar method (Hagos et al. [2]), modelling thermal discharges in water basins.

The method for the study of flow and thermal energy transport in water basins is based on Navier-Stokes equation, coupled with the temperature transport equation. 
In glaciers, convective terms are negligible (LeB et al. [3], Hutter [4], Paterson [5], Colinge and Blatter [6], Blatter et al. [7]). Therefore the problem is modelled by Stokes system, in vector, unsteady conservation form:

$$
\left\{\begin{array}{l}
\nabla \cdot \mathbf{u}=0 \\
\frac{\partial \mathbf{u}}{\partial t}-\nabla \cdot[\nu(\nabla \mathbf{u})]=-\nabla p+\mathbf{g}+\left[(\nabla \nu) \cdot(\nabla \mathbf{u})^{T}\right]
\end{array}\right.
$$

where $(\nabla \nu) \cdot(\nabla \mathbf{u})^{T}$ is a viscous forcing term.

Although an acceleration term appears in momentum equation, the final solution verifies a steady Stokes system since, at steady state, the temporal derivative vanishes.

\section{Non-dimensionalization}

Equations and boundary conditions are non-dimensionalized by means of factors defined on the basis of the velocity norm, the glacier or basin depth and the amplitude of the temperature range.

\section{Temporal evolution}

A Fractional Step technique deals with the temporal evolution. At time instant $n+1$, at first, provisional velocities are computed on the basis of a hydrostatic pressure and a surface elevation $\eta$ relative to the preceding time instant.

Subsequently the full pressure is calculated and velocities are corrected. Finally the surface elevation is computed by Saint-Venant equation and the temperature field is found.

\section{Space discretization}

The domain is subdivided into a set of cell-centred rectangular control volumes with faces orthogonal to the coordinate axes. A Finite Volume method is used to grant both local and global conservation of each physical quantity; the profiles approximating diffusive and convective terms are defined in such a way that a uniform distribution of volume dimensions is not needed. Boundary conditions are therefore approximated by Generalized Finite Difference formulae (De Biase et al. [8]). The vertical dimension of the surface volumes evolves in time.

\section{Viscosity}

The turbulent viscosity in water flows is modelled by our method in several possible ways (as an evolution of Hagos et al. [2]): constant viscosity, Prandtl method, standard $k-\varepsilon$ and low-Reynolds $k-\varepsilon$ methods (Mohammadi and Pironneau [9])

In glaciers, molecular viscosity is considered (Gudmundsson [10]). 


\section{Domain definition}

The reference system is defined in such a way that the free surface and the bedrock or bottom are, in the average, approximated by planes parallel to the $x-y$ coordinate plane, while inflow and outflow boundaries are parallel to the $y-z$ coordinate plane and lateral boundaries are parallel to the $\mathrm{x}-\mathrm{z}$ coordinate plane.

\section{The thermal energy equation in a glacier}

This equation is written as in Anderson [11], on the basis of the temperature field:

$$
\rho c \frac{D T}{D t}-\nabla \cdot(K \nabla T)=S
$$

where $\rho$ is the fluid density, $K$ is the thermal conductivity and $\mathrm{S}$, the heat production rate for unit volume, generated by ice deformation, is written on the basis of stress components, as:

$$
S=2 \mu\left[\dot{\varepsilon}_{x x}^{2}+\dot{\varepsilon}_{y y}^{2}+\dot{\varepsilon}_{z z}^{2}+2\left(\dot{\varepsilon}_{x y}+\dot{\varepsilon}_{x z}+\dot{\varepsilon}_{y z}\right)+\dot{\varepsilon}_{0}^{2}\right]=2 \mu\left[2 \dot{\varepsilon}_{\| l}+\dot{\varepsilon}_{0}^{2}\right]
$$

where $\mu$ is the dynamic viscosity and $\dot{\varepsilon}_{\| l}$ is the second invariant of the stress tensor $\varepsilon$.

\section{Case test 1: thermal discharge}

The domain for case test 1 simulates the outfall from an industrial plant: in the channel, water of a given temperature flows toward a water basin of lower temperature. The domain is a 3D rectangle of total length $20 \mathrm{~m}$, width $1 \mathrm{~m}$ and total height $2 \mathrm{~m}$, with a backward-facing step of length $10 \mathrm{~m}$ and height $1 \mathrm{~m}$; it is discretized into 10750 volumes of dimensions $\Delta x=\Delta z=0.2 \mathrm{~m}, \Delta \mathrm{y}=0.1 \mathrm{~m}$. Initial conditions are of null motion and pressure fields on the whole domain and unperturbed free surface. At the inflow boundary, homogeneous Neumann conditions for hydrodynamic pressure and for components $v, w$ of velocity are assigned, while for the $u$ component a value of $5 \mathrm{~m} / \mathrm{s}$ is given and $T=26^{\circ} C ; k=0.003 \hat{u}^{2} ; \varepsilon=c_{\mu}^{3 / 4} \frac{k^{3 / 2}}{L_{3}}$, with initial viscosity of $3 * 10^{-4} \mathrm{~m}^{2} / \mathrm{s}$.

At the outflow boundary homogeneous Neumann conditions for velocity, temperature, $k$ and $\varepsilon$ and homogeneous Dirichlet for the hydrodynamic pressure are imposed. On lateral walls friction conditions are assigned, with Chezy coefficient of $50 \mathrm{~m}^{1 / 2} / \mathrm{s}$, homogeneous Neumann conditions for temperature, $k$ and $\varepsilon$. On the free surface null stress is assumed with homogeneous Neumann conditions for temperature, $k$ and $\varepsilon$ (Barkley et al. [12], Beaudoin et al. [13]). 
146 Advanced Computational Methods in Heat Transfer IX
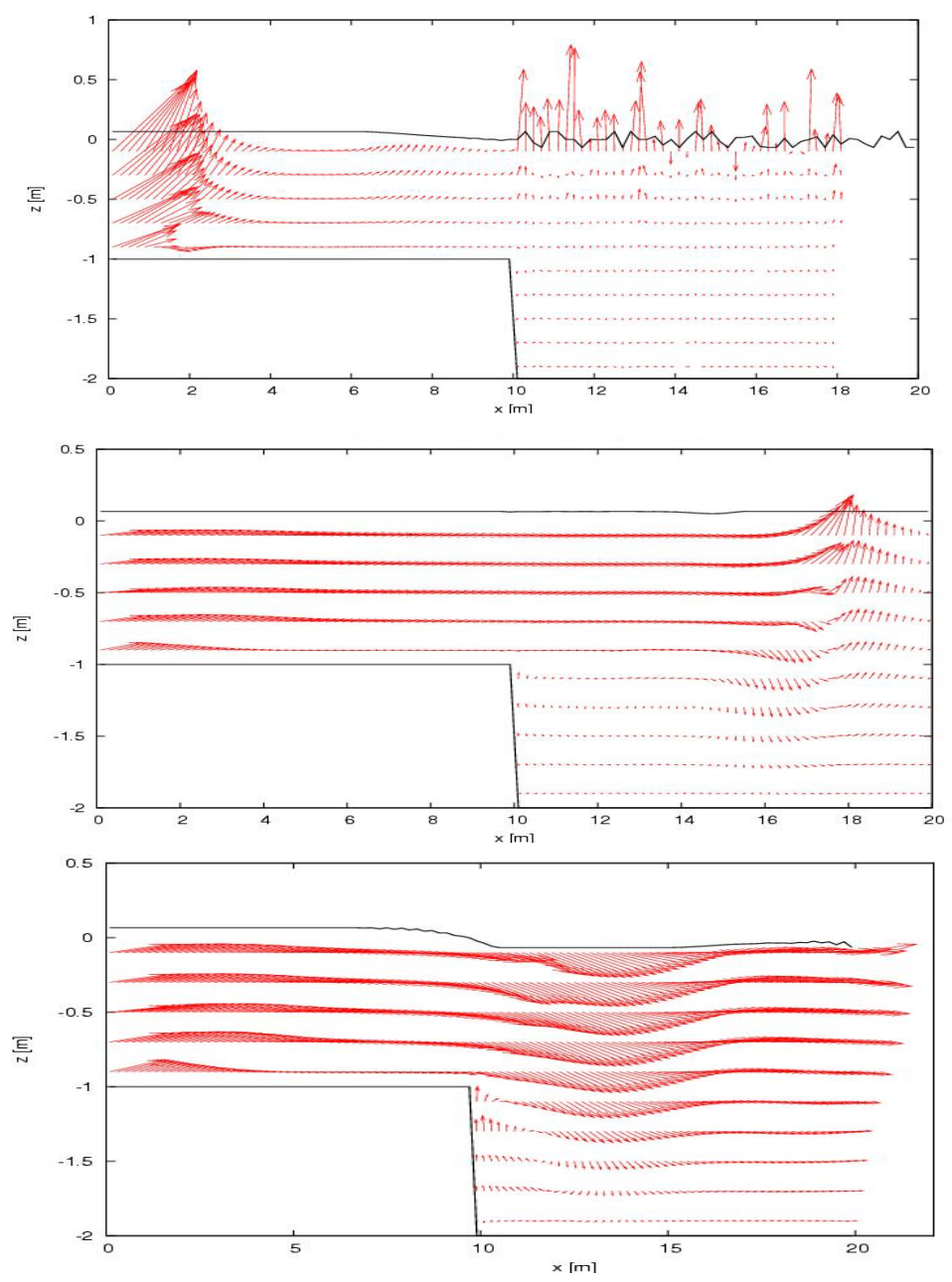

Figure 1: Motion fields with constant viscosity, Prandtl technique and a $k-\varepsilon$ method.

In figure 1 the motion fields obtained by imposing constant viscosity of $10^{-6} \mathrm{~m}^{2} / \mathrm{s}$, by Prandtl approximation and by a standard $k-\varepsilon$ method are compared.

Chezy coefficient is normally in the range between 30 and $60 \mathrm{~m}^{1 / 2} / \mathrm{s}$, depending on the substrate of the channel. Since a constant density hypothesis has been formulated in this work, the coefficient must be divided by 1000 , the water density. 

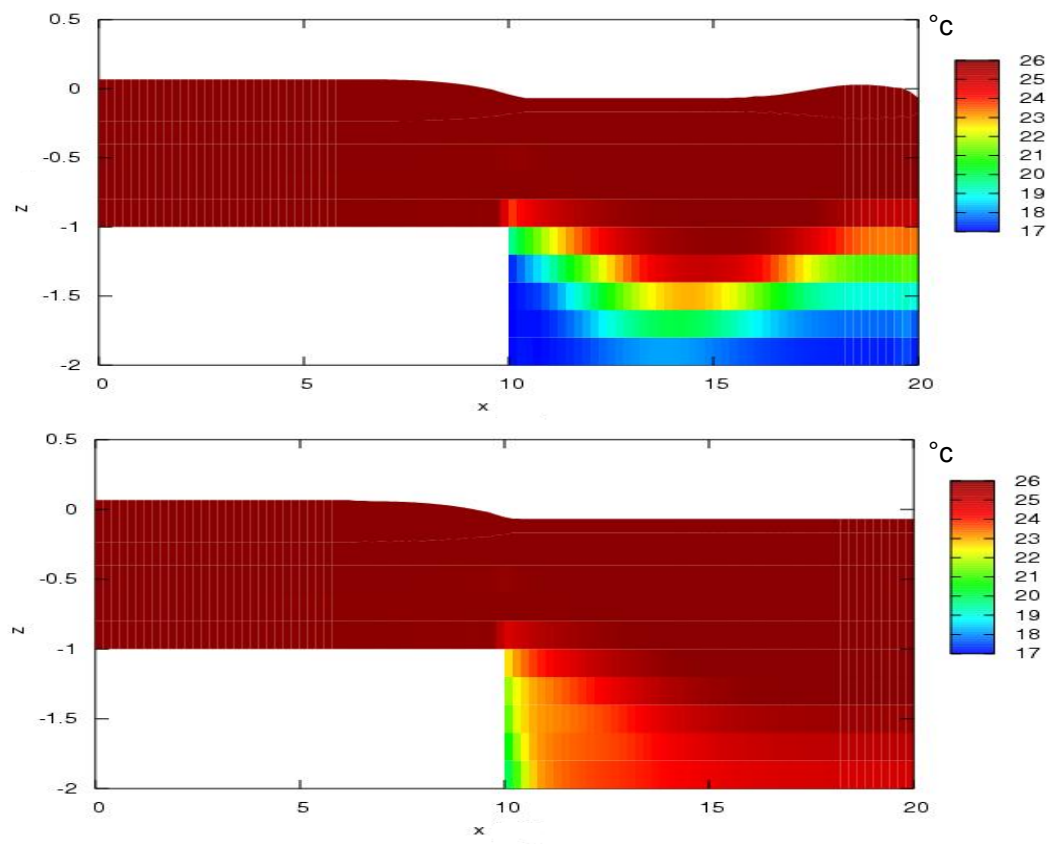

Figure 2: $\quad$ Temperature distribution after 132 and 600 time steps.

In the application presented above it is evident that the constant viscosity model is not an option for the problem at study and that the best results are obtained by means of the $k-\varepsilon$ technique.

In figure 2 the temperature distributions obtained by this technique after 132 and 600 time steps are shown.

\section{Case test 2: the Priestley Glacier}

For the glacier bedrock, the interface ice/rock is implicitly defined as

$$
B \equiv-h-z=0
$$

The kinematic boundary condition imposes the vertical velocity to equal the $\mathrm{z}$ component of the melting/freezing rate. On the lateral boundaries, orthogonal to the $y$ axis, the kinematic condition is the same, but the y component must be considered for lateral velocity; dynamic boundary conditions for velocity are: 


$$
\begin{aligned}
& u=\mathbb{C} \sigma_{x y}^{2}=\mathbb{C} \mu^{2}\left(\frac{\partial u}{\partial y}+\frac{\partial v}{\partial x}\right)^{2} \\
& v=\mathbb{C} \sigma_{y y}^{2}=\mathbb{C}\left(-P+2 \mu \frac{\partial v}{\partial y}\right)^{2} \quad \text { where } \mathbb{C} \text { is the slip coefficient. (4) } \\
& w=\mathbb{C} \sigma_{y z}^{2}=\mathbb{C} \mu^{2}\left(\frac{\partial v}{\partial z}+\frac{\partial w}{\partial y}\right)^{2}
\end{aligned}
$$

On the free surface, the kinematic boundary condition is determined by the accumulation/ablation rate vector, while dynamic B.C. are:

$$
\frac{\partial u}{\partial z}+\frac{\partial w}{\partial x}=0, \quad \frac{\partial v}{\partial z}+\frac{\partial w}{\partial y}=0, \quad \frac{\partial w}{\partial z}=0 .
$$

On the bedrock, a geothermal flux is assigned:

$$
\frac{\partial T}{\partial \mathbf{n}}=\frac{q^{g e o}}{K}
$$

where $q^{\text {geo }}$, the geothermal flux, varies between $46 \mathrm{mWm}^{-2}$ and $77 \mathrm{mWm}^{-2}$ depending on the rock age.

On lateral solid walls, at ice/ice interfaces, a null flux condition is imposed, while at rock/ice interfaces, the condition

is imposed.

$$
\frac{\partial T}{\partial y}= \pm \frac{q^{g e o}}{K}
$$

On the free surface Dirichlet conditions can be assigned (Paterson [5], Luthi and Funk $[14,15]$ ), or a Neumann condition of the form (Hutter, [4]):

$$
\frac{\partial T}{\partial \mathbf{n}}=\frac{q^{\text {atm }}}{K}
$$

where $q^{\text {atm }}$ is the atmospheric heat flux.

$$
\frac{T^{n+1}-T^{n}}{\Delta t}+\nabla \cdot\left[u_{i}^{n+1} T^{n+1}-\kappa\left(\frac{\partial T}{\partial x_{i}}\right)^{n+1}\right]=\frac{S^{n+1}}{c}, \mathrm{i}=1,2,3
$$

where $S$ is the heat production term, $\kappa$ is the thermal diffusivity and $c$ is the thermal capacity.

The area of study, built by means of the RAMP database and of aerial images, has dimensions $\mathrm{L}=14000 \mathrm{~m}, \mathrm{D}=7000 \mathrm{~m}$ and $\mathrm{H}=1000 \mathrm{~m}$, discretized into 5466 volumes. Dimensions of internal control volumes are: $\Delta x=\Delta y=600 \mathrm{~m}$ and $\Delta z=100 \mathrm{~m}$. Components of the gravity acceleration vector are: $g_{x}=0.169 \mathrm{~m} / \mathrm{s}^{2}$, $\mathrm{g}_{\mathrm{y}}=0.007 \mathrm{~m} / \mathrm{s}^{2}$ and $\mathrm{g}_{\mathrm{z}}=9.909 \mathrm{~m} / \mathrm{s}^{2}$. Homogeneous Neumann conditions are imposed for velocity and pressure at the inflow boundary, free exit for velocity and homogeneous Neumann for pressure are assigned at the outflow boundary. The free surface can evolve in space and time. On the bedrock and on the lateral 
walls a slip condition is imposed, with slip coefficients $\mathbb{C}=5 \times 10^{-18} \mathrm{~ms}^{-1} \mathrm{~Pa}^{-2}$ and $\mathbb{C}=10^{-18} \mathrm{~ms}^{-1} \mathrm{~Pa}^{-2}$, respectively. The variable viscosity is computed by Glen's constitutive law, with parameter $\mathrm{n}=3$ (Pettit and Waddington [16]). Initial conditions are null motion and pressure fields on the entire domain.
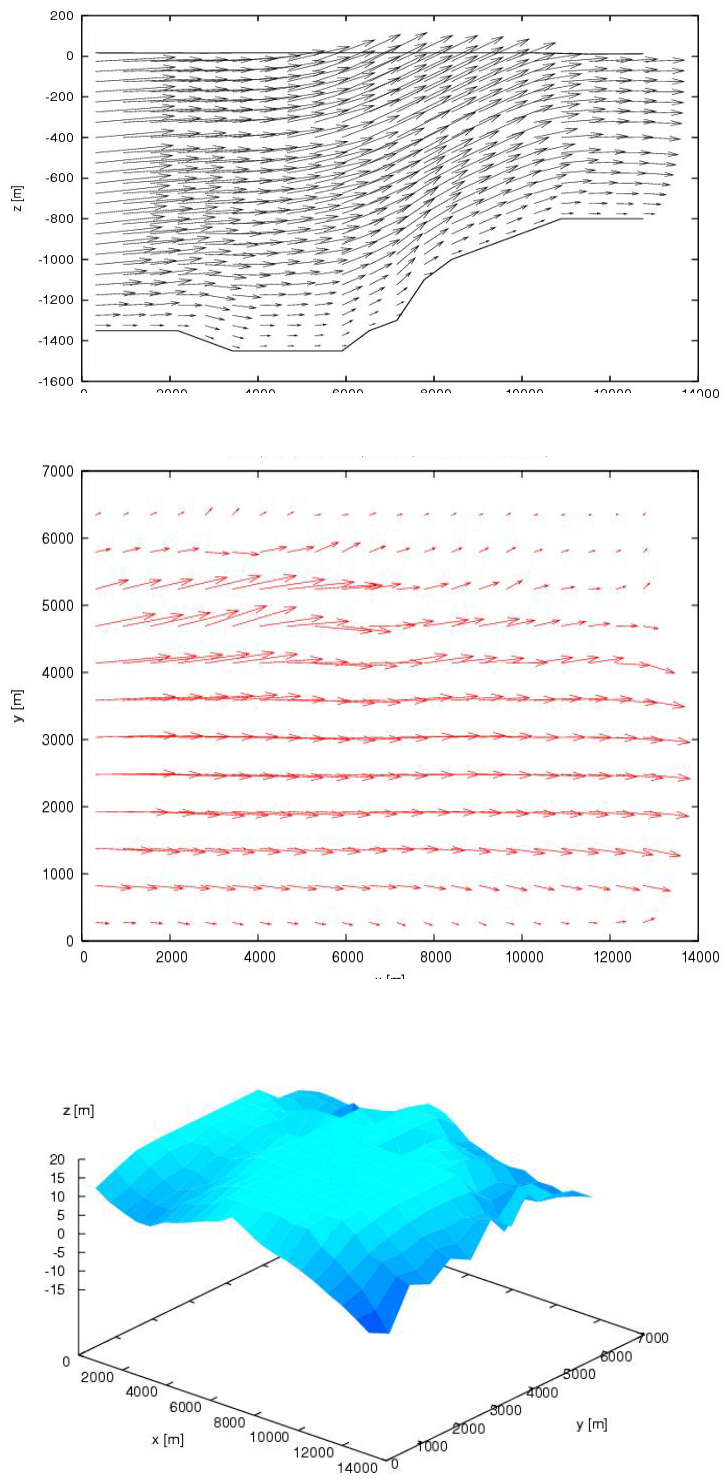

Figure 3: Velocity field in a longitudinal section and a horizontal section; surface elevation in the glacier. 

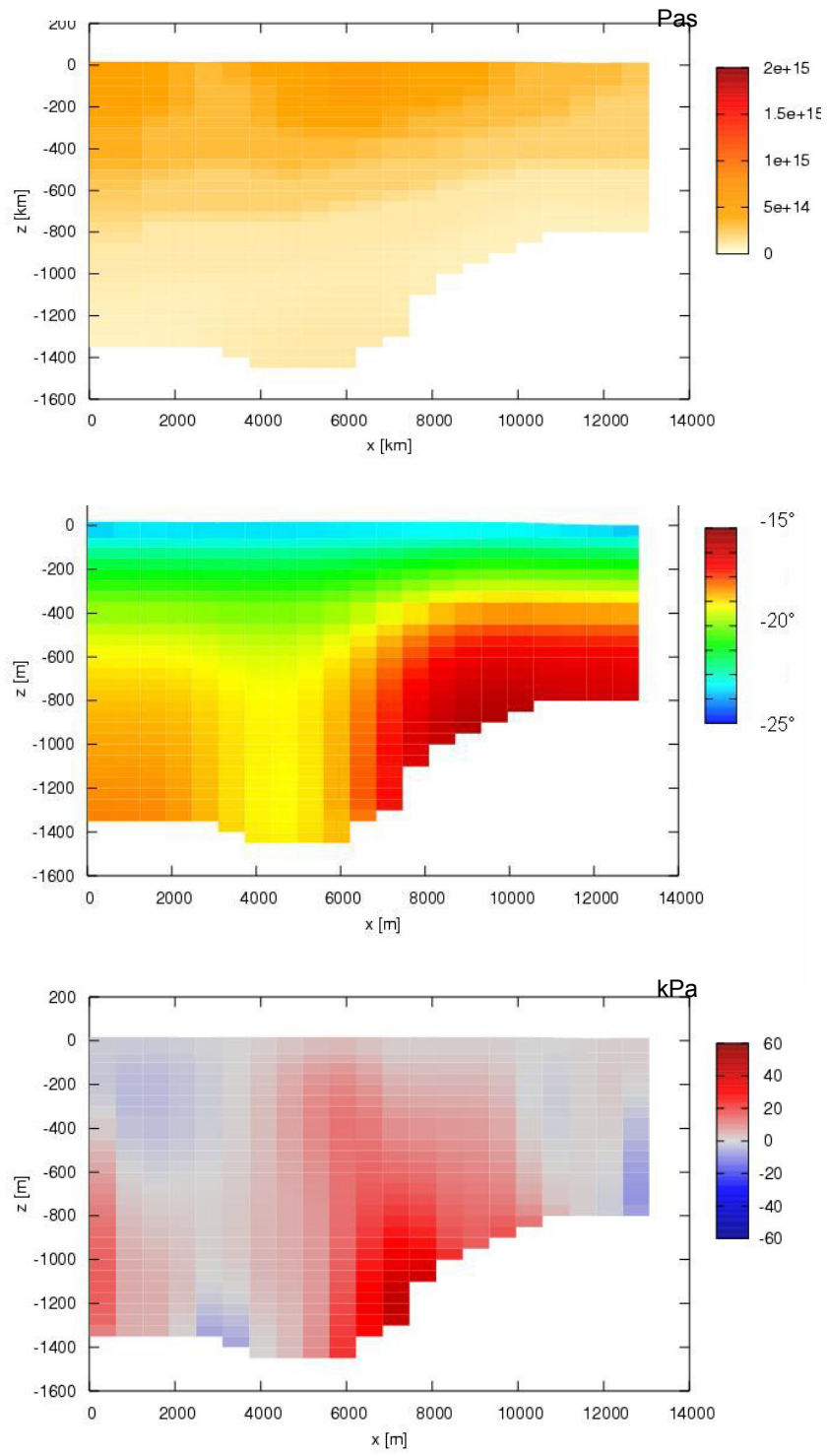

Figure 4: Viscosity field, temperature distribution and hydrodynamic pressure in a longitudinal section of the Priestley Glacier. 


\section{Conclusion}

Results presented in this paper are in full agreement with the theory and with physical observations and measurements (Barkley et al. [12], Beaudoin et al. [13] for water applications, Kamb [17], Pattyn [18] for the Priestley application).

In future some more work should be done in order to allow a more flexible assignment of boundary conditions on the volumes belonging to two different boundary surfaces. Indeed the rectangular shape of the volumes creates a steplike border of the computational domain and this makes it difficult to follow irregularities of the physical domain and to assign boundary conditions on some volume faces.

\section{References}

[1] Deponti A., Pennati V., De Biase L., A fully 3D finite volume method for incompressible Navier-Stokes equations, Int. J. for Numerical Methods in Fluids, (in press; available online in Wiley Interscience, DOI: 10.1002/fld.1190), 2006

[2] Hagos S., Deponti A., Pennati V., De Biase L., Applicazione di un nuovo metodo ai volumi finiti a problemi di inquinamento termico, La Termotecnica, Aprile 2005

[3] LeB. Hooke R., Principles of Glacier Mechanics, Prentice Hall, Upper Saddle River, New Jersey 07458, 1998.

[4] Hutter K., Theoretical Glaciology, Material Science of Ice and the Mechanics of Glaciers and Ice Sheets, D. Reidel Publishing Company, Dordrecht, Holland, 1983.

[5] Paterson W.S.B., The Physics of Glaciers, Third Edition, Pergamon, 1994.

[6] Colinge J., Blatter H., Stress and Velocity fields in glaciers: Part I. Finitedifference schemes for higher-order glacier models, Journal of Glaciology, Vol. 44, N. 148, pages 448-456 (a), 1998.

[7] Blatter H., Clarke G. K. C., Colinge J., Stress and Velocity fields in glaciers: Part II. Sliding and basal stress distribution, Journal of Glaciology, Vol. 44, N. 148, pages 457-466 (b), 1998.

[8] De Biase L., Feraudi F., Pennati V., A Finite Volume Method for the solution of Convection-Diffusion 2D problems by a Quadratic Profile with Smoothing, International Journal of Numerical Methods for Heat \& Fluid Flow, Vol. 6, N. 4, pages 3-24, 1996.

[9] Mohammadi B., Pironneau O., Analysis of the K-Epsilon Turbulence Model, John Whiley and Sons, 1994.

[10] Gudmundsson G. H., Basal-flow characteristics of a non-linear flow sliding frictionless over strongly undulations bedrock, Journal of Glaciology, Vol. 43, N. 143, pages. 80-89 (b) 1997.

[11] Anderson J.D., Introduction, Von Karman Institute for Fluids Dynamics Lecture Series: Introduction to Computational Fluid Dynamics, pages 18-22, 1983 
[12] Barkley D., Gomes M., Henderson R.D., Three-dimensional instability in Flow over a Backward Facing Step. International Journal for Fluid Mechanic, Vol. 473, pages 167-190, 2002.

[13] Beaudoin J.F., Cadot O., Aider J.L., Centrifugal Instability in the Backward Facing Step, International Journal for Fluid Mechanic, 2003.

[14] Lüthi M., Funk M., Dating ice cores from a high Alpine glacier with a flow model for cold firn, Annals of Glaciology N. 31, pages 69-79, 2000.

[15] Lüthi M., Funk M., Modelling heat flow in a cold, high-altitude glacier: interpretation of measurements from Colle Gnifetti, Swiss Alps, Journal of Glaciology, N. 47(157), pages 314-324, 2001.

[16] Pettit E.C., Waddington E.D., Ice flow at low deviatoric stress, Journal of Glaciology, vol. 49, N. 166, pages. 359-369, 2003

[17] Kamb B., Sliding motion of glaciers: Theory and observation, Reviews of Geophysics and Space Physics, N. 8, 4, pages 673-728, 1970.

[18] Pattyn F., Ice-sheet modelling at different spatial resolutions: focus on the grounding zone, Annals of Glaciology, N. 31, pages 211-216, 2000. 